\title{
The Role of Oxygen Abundances in Exoplanet Host Star C/O Ratios: A Case Study of 55 Cnc
}

\author{
Johanna K. Teske ${ }^{1}$, Katia Cunha ${ }^{2}$, Verne V. Smith ${ }^{3}$, \\ Simon C. Schuler ${ }^{4}$ and Caitlin A. Griffith ${ }^{5}$ \\ ${ }^{1}$ Steward Observatory, University of Arizona, email: jteske@as.arizona.edu \\ ${ }^{2}$ Observatório Nacional, Brazil \\ ${ }^{3}$ National Optical Astronomy Observatory \\ ${ }^{4}$ University of Tampa \\ ${ }^{5}$ Lunar and Planetary Laboratory, University of Arizona
}

\begin{abstract}
We derive the $\mathrm{C} / \mathrm{O}$ ratio for the exoplanet host star $55 \mathrm{Cnc}$ based on archive Keck/HIRES spectra. The $\mathrm{C} / \mathrm{O}$ ratio varies widely depending on which oxygen abundance indicator - the $6300.3 \AA[\mathrm{O}$ I] line, the $6363.8 \AA$ [O I] line, or the $7774 \AA$ O I triplet - is used, and we find there is still a large uncertainty even based on individual abundance indicators. This case study demonstrates that caution and care are necessary when determining exoplanet host star $\mathrm{C} / \mathrm{O}$ ratios, and when considering host star $\mathrm{C} / \mathrm{O}$ ratios in inferring exoplanet compositions.
\end{abstract}

Keywords. planetary systems: formation - stars: abundances - stars: individual ( 55 Cnc)

\section{Motivation}

Precise Mass and radius measurements of super-Earths can be used to constrain composition by comparing them to various interior models. One such case is the transiting super-Earth 55 Cnc e, part of a 5-planet system originally discovered through RV observations (e.g., McArthur et al. 2004). Using the most recent mass and radius measurements, Madhusudhan et al. (2012) report that 55 Cnc e could have a C-rich interior. This in contrast to previous modeling of $55 \mathrm{Cnc}$ e, which suggests an interior of Fe and silicates below a super-critical water envelope by assuming a solar-like, O-rich interior (e.g., Gillon et al. 2012). Madhusudhan et al. (2012) assume that the host star 55 Cnc is C-rich, based on the $\mathrm{C} / \mathrm{O}_{55 \mathrm{Cnc}}=1.12 \pm 0.19$ of Delgado Mena et al. (2010). Given the weight of this assumption in current theories of 55 Cnc e's interior composition, and recent work that questions previously-measured C/O ratios (e.g. Nissen 2013; Fortney 2012), we examine further the $\mathrm{C} / \mathrm{O}$ ratio of $55 \mathrm{Cnc}$ and show it is not so easily constrained.

\section{Methods}

We used archive Keck/HIRES high-resolution echelle spectroscopy of 55 Cnc (PI: Shkolnik) to first derive 55 Cnc's basic stellar parameters following classical/standard techniques. Abundances were then determined differentially by either measuring equivalent widths or using the spectral synthesis technique in $55 \mathrm{Cnc}$ and in the Sun using the archive Keck/HIRES spectrum of Vesta (PI: Marcy). 


\section{Results}

$6300 \AA[O$ I] line (synthesis). The [O I] $6300.30 \AA$ line gives the lowest oxygen abundance, resulting in $\mathrm{C} / \mathrm{O} \sim 0.97$. This line is blended with a Ni I line that becomes more important at higher metallicities, for which most high $\mathrm{C} / \mathrm{O}$ values have been found (Nissen 2013). When determining $[\mathrm{O} / \mathrm{H}]$, we assumed the $\mathrm{Ni}$ abundance measured directly from $55 \mathrm{Cnc}$, but by changing $[\mathrm{Ni} / \mathrm{H}]$ within our derived error $( \pm 0.05)$, the $\mathrm{C} / \mathrm{O}$ ratio varies from $\sim 0.54-1.4$. With the errors determined for $\mathrm{C}$ and this $\mathrm{O}$ line, assuming the $\mathrm{A}(\mathrm{Ni})=6.68$ value, the $\mathrm{C} / \mathrm{O}$ ratio ranges from $\sim 0.5-1.3$.

O I triplet with NLTE. The O I triplet is known to suffer from NLTE effects (e.g. Kiselman 2001), for which several groups have published corrections. For varying NLTE corrections (Takeda 2003; Ramírez et al. 2007; Fabbian et al. 2009), C/O ranges from 0.560.79 , with an error $\sim 0.2$ based on the LTE abundances. However, Schuler et al. (2006) pointed out that such NLTE corrections are not reliable for stars as cool as 55 Cnc $\left(T_{\text {eff }} \leqslant 5450 \mathrm{~K}\right)$, which show an increase with decreasing $T_{\text {eff }}$, in direct contrast to canonical NLTE calculations.

$6363 \AA[O$ I] line (synthesis). The [O I] $6363.78 \AA$ line gives a $\mathrm{C} / \mathrm{O} \sim 0.8$. It is blended with a CN line; while Caffau et al. (2013) finds with 3D models that the CN contribution to this line is $<20 \%, \mathrm{CN}$ contributes more in the cooler $55 \mathrm{Cnc}(\sim 30-40 \%)$. Moreover, Caffau et al. (2013) show that in dwarf stars this line give larger oxygen abundances than the [O I] $6300.3 \AA$ line, although the origin of this discrepancy is still unclear. Again, within the $\mathrm{C}$ and $\mathrm{O}$ errors (for this line specifically), C/O ranges from $\sim 0.48-1.06$.

\section{Conclusions}

While previous measurements indicated the $\mathrm{C} / \mathrm{O}$ ratio of $55 \mathrm{Cnc}$ to be $\sim 1$, we show here that the picture is not so clear. This calls into question the prediction of Madhusudhan et al. (2012) that the small-mass exoplanet 55 Cnc e is C-rich, which was based firmly on the star also being C-rich. Measuring $\mathrm{O}$ is difficult in solar-type stars, and becomes even more complicated in cooler, metal-rich stars like 55 Cnc. Our case study demonstrates the extreme caution that must be used when determining exoplanet host star (and any star's) $\mathrm{C} / \mathrm{O}$ ratios, particularly the sensitivity of all three major oxygen abundance indicators to different effects that are not always easy to account for and changes based on stellar parameters.

\section{References}

Borucki, W. J., et al. 2011, ApJ, 736, 19

Caffau, E., et al. 2013, A\&A A, 554, 126

Caffau, E., et al. 2008, AAP, 488, 1031

Delgado Mena, E., et al. 2010, ApJ, 725, 2349

Fabbian, D., et al. 2009, AAP, 500, 1221

Fortney, J. J. 2012, ApJL, 747, L27

Gillon, M., et al. 2012, AAP, 539, A28

Kiselman, D. 2001, NAR, 45, 559

Madhusudhan, N., et al. 2012, ApJL, 759, L40

McArthur, B. E., et al. 2004, ApJL, 614, L81

Nissen, P. E. 2013, AAP, 552, A73

Ramírez, I., et al. 2007, AAP, 465, 271

Schuler, S. C., et al. 2006, ApJ, 636, 432

Takeda, Y. 2003, AAP, 402, 343 